\title{
Preventive effects of citrulline on Western diet-induced non-alcoholic fatty liver disease in rats
}

\author{
Prasanthi Jegatheesan ${ }^{1}$, Stéphanie Beutheu ${ }^{1}$, Kim Freese ${ }^{1}$, Anne-Judith Waligora-Dupriet ${ }^{2}$, \\ Esther Nubret ${ }^{1}$, Marie-Jo Butel ${ }^{2}$, Ina Bergheim ${ }^{3}$ and Jean-Pascal De Bandt ${ }^{1,4_{*}}$ \\ ${ }^{1}$ EA4466, Faculty of Pharmacy, Paris Descartes University, Sorbonne Paris Cité, 75006 Paris, France \\ ${ }^{2}$ EA4065, Faculty of Pharmacy, Paris Descartes University, Sorbonne Paris Cité, 75006 Paris, France \\ ${ }^{3}$ Institute of Nutritional Science, SD Model Systems of Molecular Nutrition, Friedrich-Schiller University Jena, O7743 Jena, Germany \\ ${ }^{4}$ Clinical Chemistry Department, Hôpitaux Universitaires Paris Centre, APHP, 75679 Paris, France
}

(Submitted 30 December 2015 - Final revision received 22 March 2016 - Accepted 5 April 2016)

\section{Abstract}

A Western diet induces insulin resistance, liver steatosis (non-alcoholic fatty liver disease (NAFLD)) and intestinal dysbiosis, leading to increased gut permeability and bacterial translocation, thus contributing to the progression of NAFLD to non-alcoholic steatohepatitis. In the present study, we sought, in a model of Western diet-induced NAFLD, to determine whether citrulline (Cit), an amino acid that regulates protein and energy metabolism, could decrease Western diet-induced liver injuries, as well as the mechanisms involved. Sprague-Dawley rats were fed a high-fat diet ( $45 \%)$ and fructose $(30 \%)$ in drinking water or a control diet associated with water (group C) for 8 weeks. The high-fat, high-fructose diet (Western diet) was fed either alone (group WD) or with Cit ( $1 \mathrm{~g} / \mathrm{kg}$ per d) (group WDC) or an isonitrogenous amount of non-essential amino acids (group WDA). We evaluated nutritional and metabolic status, liver function, intestinal barrier function, gut microbiota and splanchnic inflammatory status. Cit led to a lower level of hepatic TAG restricted to microvesicular lipid droplets and to a lower mRNA expression of CCAAT-enhancer-binding protein homologous protein, a marker of endoplasmic reticulum stress, of pro-inflammatory cytokines $I l 6(P<0 \cdot 05)$ and Tnf $\alpha$, and of toll-like receptor 4 (Tlr 4$)(P<0.05)$. Cit also improved plasma TAG and insulin levels. In the colon, it decreased inflammation (Tnfo and Tlr4 expressions) and increased claudin-1 protein expression. This was associated with higher levels of Bacteroides/Prevotella compared with rats fed the Western diet alone. Cit improves Western diet-induced liver injuries via decreased lipid deposition, increased insulin sensitivity, lower inflammatory process and preserved antioxidant status. This may be related in part to its protective effects at the gut level.

\section{Key words: Non-essential amino acids: Non-alcoholic steatohepatitis: Liver: Microbiota: Gut permeability}

Non-alcoholic fatty liver disease (NAFLD) has evolved as the most common form of chronic liver disease, and has become a major public health issue as it increasingly appears as a systemic disease in the context of the metabolic syndrome ${ }^{(1)}$. Hepatic fat accumulation induces not only cellular stresses such as oxidative stress and endoplasmic reticulum stress but also insulin resistance (IR) and inflammation, which lead to the development of non-alcoholic steatohepatitis $(\mathrm{NASH})^{(2,3)}$. Visceral fat accumulation is regarded as a significant risk factor for the development of NAFLD and its progression to $\mathrm{NASH}^{(4)}$. NASH can further deteriorate into cirrhosis and hepatocellular carcinoma $^{(2)}$. Energy imbalance and an 'unhealthy' dietary pattern have been claimed to be key factors in the development and progression of steatosis to $\mathrm{NASH}^{(5)}$. High-fat, high-fructose diets, also called Western diets, have been shown to induce IR and de novo lipogenesis in the liver with further deterioration in liver function ${ }^{(6)}$. Several recent studies have also pointed to alterations in intestinal microbiota and gut barrier function as important contributors to the development of $\mathrm{NASH}^{(7,8)}$. The composition of intestinal microbiota in NASH patients is characterised by a higher level of Clostridium coccoides and a lower level of Bacteroides/Prevotella ${ }^{(4)}$. Dysbiosis in turn alters gut permeability and increases bacterial translocation, which results in endotoxin-induced activation of toll-like receptor 4 (TLR4) in the liver, leading to the production of proinflammatory cytokines ${ }^{(9)}$. Thus, the intestinal environment may be at least as important as hepatic metabolic stress in the progression of the disease ${ }^{(4)}$.

Besides lifestyle modifications (weight loss, reduction in dietary fat and sweetened-drink consumption, etc.), which are

Abbreviations: AA, amino acid; Arg, arginine; ChREBP, carbohydrate-responsive element-binding protein; Cit, citrulline; Gln, glutamine; IR, insulin resistance; MPO, myeloperoxidase; NAFLD, non-alcoholic fatty liver disease; NASH, non-alcoholic steatohepatitis; NEAA, non-essential amino acids; TLR4, toll-like receptor 4; WD, Western diet; WDA, Western diet + non-essential amino acids; WDC, Western diet + citrulline.

* Corresponding author: Professor J.-P. De Bandt, fax +33 153739 952, email jean-pascal.de-bandt@parisdescartes.fr 
difficult to implement, or vitamin E treatment, for which there is a safety concern in terms of cancer risk, effective treatment strategies are limited ${ }^{(10)}$, probably because $\mathrm{NASH}$ is a multifactorial disease. Amino acid (AA) or protein supplementations may offer a useful alternative, as they may target several mechanisms involved in disease progression such as steatosis and IR, as well as dysbiosis and altered gut function. They have been shown to improve steatosis via their effects on lipid metabolism, insulin sensitivity and glucose tolerance ${ }^{(11,12)}$. The mechanism proposed may be (i) decreased de novo lipogenesis through down-regulation of genes involved in lipogenesis, (ii) increased lipid oxidation or (iii) modulation of lipid-induced inflammation. However, although protein supplementation has been shown to improve gut function and microbiota equilibrium ${ }^{(13,14)}$, its effectiveness in terms of gut/ liver interactions remains to be demonstrated. Recent studies emphasise the importance of specific AA such as citrulline (Cit) in this setting ${ }^{(15-17)}$. From a metabolic point of view, we previously showed that Cit improves hepatic lipid metabolism, insulin sensitivity and steatosis in a model of fructose-induced NAFLD $^{(18,19)}$ where gut function is preserved. Moreover, Cit supplementation is associated with an improvement in plasma AA and lean body mass suggesting improved protein metabolism. Interestingly, Cit may also be protective at the gut level and on gut/liver interactions. Indeed, Cit attenuates mucosal damage and improves intestinal permeability in a model of 5-fluorouracil-induced mucositis in mice. Recently, it has also been shown that Cit mitigates intestinal inflammation $^{(17)}$ and promotes intestinal adaptation to massive intestinal resection ${ }^{(20)}$. Finally, as already shown for arginine (Arg), its precursor/derivative, which regulates intestinal microbiota ${ }^{(21)}$, Cit may also possibly affect microbiota composition in the large intestine. This led us to wonder about the potential interest of $\mathrm{Cit}$ in the prevention of the disease aggravation into NASH.

This present study's hypothesis was that Cit might improve some of the factors involved in disease progression not only at the liver but also the gut and peripheral organ level.

The aims of this study were thus to assess the effects of oral Cit administration on liver and gut function in a rat model of NAFLD induced by an 8-week Western diet and to elucidate the mechanisms involved.

\section{Methods \\ Animals}

In total, forty-three 6-week-old male Sprague-Dawley rats (190-220 g) were purchased from Charles River. They were housed individually in a temperature-controlled room with a $12 \mathrm{~h}$ light-12 h dark cycle. They were given free access to water and standard rodent chow (UAR A04; SAFE) ad libitum for a 1-week acclimatisation period.

For the feeding experiments, the rats received either standard rodent chow (UAR A04) or a Western diet combining a $45 \%$ fat diet (824053; Special Diets Service) with 30\% fructose in drinking water (F0127; Sigma-Aldrich). Compositions of the diets are given in the online Supplementary Table S1.
Animal care and experimentation complied with both French and European Community regulations for animal care and experimentation. All the procedures were conducted in accordance with the guidelines for animal care of the regional ethics committee (Comité régional d'éthique en matière d'expérimentation animale, Ile-de-France), and the study protocol was approved by the same committee (registration no. 00737.02).

\section{Experimental design}

The rats were randomly assigned to four groups (10-13 per group) to receive for 8 weeks either standard rodent chow ( $\mathrm{C}$ group) or a Western diet either alone (WD group) or supplemented with non-essential amino acids (NEAA, WDA group) or with Cit $1 \mathrm{~g} / \mathrm{kg}$ per d (WDC group). The NEAA supplement contained isomolar amounts of six NEAA (alanine, glycine, proline, aspartate, histidine and serine) and was isonitrogenous to the Cit supplement. The animals received their respective food and drinks ad libitum. Body weight (BW) was measured at the beginning of the experiment and then once a week throughout the study. In addition, once a week throughout the study, food and drink intakes were monitored, $24 \mathrm{~h}$ urine samples were collected and $\mathrm{N}$ excretion was measured by pyrochemiluminescence (Antek 9000; Antek). At the end of the feeding period and after an overnight fast, the rats were anaesthetised by isoflurane inhalation. Portal and aortal blood samples were taken, and the rats were euthanatised by exsanguination for liver and gut sample collection as described previously ${ }^{(18)}$. Body composition, particularly localisation and size of fat deposits, was determined by dissection and weighing. To study gut microbiota, samples of caecal content and colon mucosa were collected and frozen at $-80^{\circ} \mathrm{C}$ until analysis.

\section{Metabolic assessment}

For AA analysis, plasma samples were de-proteinised with $30 \%$ $(\mathrm{w} / \mathrm{v})$ sulphosalicylic acid solution, separated and quantified by ion-exchange chromatography with post-column ninhydrin derivatisation using a JLC-500/V AminoTac ${ }^{\mathrm{TM}}$ analyzer (Jeol Ltd). Plasma liver enzyme activities (alanine aminotransferase (ALT), aspartate aminotransferase (AST), alkaline phosphatase (ALP)) and plasma levels of bilirubin, total cholesterol, TAG, glucose and uric acid were determined using standard techniques on a multi-parameter analyzer (Cobas C 6000; Roche).

Plasma insulin was measured by ELISA, using a commercial kit (Insulin Rat Ultrasensitive ELISA; ALPCO), following the manufacturer's protocol. Insulin sensitivity was evaluated using the homoeostasis model assessment of insulin resistance (HOMA-IR): (fasted insulin $(\mathrm{mU} / \mathrm{l}) \times$ fasted glucose $(\mathrm{mm})) / 22.5$.

\section{Hepatic steatosis and inflammation}

Frozen liver tissue samples were homogenised in 5\% NP-40 to extract lipids. Hepatic TAG content was assessed using a commercially available TAG quantification kit (Abcam).

For histological evaluation of hepatic lipid accumulation, frozen liver sections $(10 \mu \mathrm{m})$ were fixed with $10 \%$ formalin in 
PBS for $30 \mathrm{~min}$, stained with Oil Red O (Sigma-Aldrich) for $30 \mathrm{~min}$ and then washed four times with sterile water. Representative photomicrographs were captured at a $20 \times$ magnification using a camera-equipped microscope (DM 4000B, Leica Microsystems CMS GmbH).

Liver samples were homogenised in (ten times their volume) $10 \%$ TCA-0.5 mm-EDTA, using an Ultra Turrax blender (IKA-Labortechnik). GSH and GSSG level was determined in the supernatant by reverse-phase HPLC coupled with MS (Thermo Fisher Scientific).

\section{RNA isolation and real-time PCR}

Total RNA from liver and colon mucosa samples was extracted with TRIzol reagent (Invitrogen), and complementary DNA was synthesised using the QuantiTect Reverse Transcription kit (Qiagen). Real-time PCR was performed using the QuantiTect SYBR-Green PCR kit (Qiagen) according to the manufacturer's instructions. To check for variations in the reactions, all PCR data were normalised against $\beta$-actin expression. PCR primers for Tlr4, carbohydrate-responsive element-binding protein (Chrebp), microsomal TAG transfer protein (Mtp), Tnfo, Il6 and $\mathrm{C} / \mathrm{EBP}$ homologous protein (Chop) are described in the online Supplementary Table S2.

The comparative $C_{t}$ method was used to determine the amount of target gene expression, normalised to an endogenous reference gene and relative to a calibrator $\left(2^{-\Delta \Delta^{C_{t}}}\right)$.

\section{Determination of endotoxin levels and intestinal inflammatory status}

Portal plasma samples were heated at $75^{\circ} \mathrm{C}$ for $5 \mathrm{~min}$. Plasma endotoxin levels were determined using a commercially-available end point Limulus Amebocyte Lysate assay (Charles River) with a concentration range of $0.005-0.5 \mathrm{EU} / \mathrm{ml}$

Myeloperoxidase (MPO) activity, a marker of polymorphonuclear infiltration, was determined in colon mucosa as described by Barone et al. ${ }^{(22)}$. In brief, colon mucosa was homogenised in $50 \mathrm{mmol} / \mathrm{l}$ potassium phosphate buffer at $\mathrm{pH} 6$ containing $0.5 \%$ hexadecyltrimethyl ammonium bromide. The homogenates were centrifuged for $30 \mathrm{~min}$ at $12500 \mathrm{~g}$ and $4^{\circ} \mathrm{C}$. The supernatants were collected and incubated with $0.167 \mathrm{mg} / \mathrm{ml} \mathrm{o-dianisidine}$ dihydrochloride (Sigma-Aldrich) and $0.0005 \% \mathrm{H}_{2} \mathrm{O}_{2}$ in $50 \mathrm{mmol} / \mathrm{l}$ $\mathrm{PBS}$ at $\mathrm{pH}$ 6. The measurements were made at $460 \mathrm{~nm}$ using a microplate reader (MRX; Dynex Technologie) in duplicate; 1 unit of MPO activity was defined as the quantity of enzyme hydrolysing $1 \mathrm{mmol}$ of $\mathrm{H}_{2} \mathrm{O}_{2} / \mathrm{min}$.

\section{Intestinal tight-junction protein expression}

Colon mucosa was homogenised in lysis buffer ( 50 mм-Hepes, $150 \mathrm{~mm}-\mathrm{NaCl}, 10 \mathrm{~mm}$-EDTA, $10 \mathrm{~mm}-\beta$-glycerophosphate, $100 \mathrm{~mm}$ NaF, 2 mm-orthovanadate, $1 \%$ Triton X-100, anti-protease and phosphatase inhibitor cocktail). After centrifugation for $30 \mathrm{~min}$ at $13000 \mathrm{~g}$ and $4^{\circ} \mathrm{C}$, the supernatants were collected. Protein levels were determined using the bicinchoninic acid assay (Protein Assay kit; Interchim). Claudin-1, zonula occludens 1 (ZO-1) and glyceraldehyde 3-phosphate dehydrogenase (GAPDH) expressions were determined according to the technique previously described $^{(23)}$ using primary antibodies: rabbit anti-claudin-1 (1:1000; Invitrogen), rat anti-ZO-1 (1:500; Millipore) and rabbit anti-GAPDH (1:2000; Cell Signaling). After three washes with TBS-1\% Tween 20 buffer, the membranes were incubated with peroxidase-conjugated goat anti-rabbit or anti-rat IgG (Dakocytomation) for $1 \mathrm{~h}$ at room temperature and revealed using the enhanced chemiluminescence detection system (GE Healthcare). Protein bands were quantified by densitometry using a CCD camera (ImageQuant LAS 4000; GE Healthcare) and ImageQuant TL software (GE Healthcare). Protein expression levels were normalised to GAPDH.

\section{Caecal and mucosa-associated microbiota analysis}

For mucosa-associated microbiota analysis, colons were flushed with sterile PBS, and the mucosa was scraped. Caecal microbiota and mucosa-associated total bacteria and main bacterial groups were quantified using real-time PCR. Total DNA was extracted using guanidium isothiocyanate and the mechanical bead beating method as previously described ${ }^{(24)}$. Total bacteria populations, Clostridium leptum group, Bacteroides/Prevotella group and Bifidobacterium, were quantified using TaqMan ${ }^{\circledR}$ qPCR. C. coccoides group, enterococci, Lactobacillus/Leuconostoc/ Pediococcus group and Akkermansia muciniphila were quantified using SYBR-Green ${ }^{\circledR}$ qPCR. The primers and probes used are described in the online Supplementary Table S3. When a species or bacterial group was not detected, a value of $3 \log _{10}$ colonyforming units/g of mucosa - that is, corresponding to about half the detection limit - was used for statistical analysis.

\section{Data analysis}

Results are expressed as mean values and standard deviations. Statistical analysis was performed using Prism 6.0 $\left(\mathrm{GraphPad}^{\circledR}\right.$ software). Homogeneity of variance of the data was assessed using Bartlett's test. Differences between groups were analysed using ANOVA followed by Fisher's protected least significant difference test. In case of heterogeneity of variance, a KruskallWallis test followed by Dunn's test was performed. For daily food intake and BW, ANOVA for repeated measurement was used, and differences were analysed using Bonferroni's test. For all the tests, $P<0.05$ was considered significant.

\section{Results}

Effect of diet on food intake, body weight and adiposity (Table 1)

BW did not differ among groups. Although food intake was lower in all the Western diet-fed rats $(P<0.05)$, total energy intake was similar in the four groups. However, there were significant differences in body composition: Western diet resulted in higher visceral fat in WD $(P=0.003)$ and WDA $(P=0.002)$ groups than in controls and in lower lean mass $(P<0.05)$. In rats fed Western diet supplemented with Cit, the gain in visceral fat mass was lower (C $v$. WDC: NS) and the loss 
Table 1. Body composition and food intake of rats fed a control diet (C; $n 10)$, a Western diet (WD; $n$ 13), a Western diet and non-essential amino acids (WDA; $n$ 10) or a Western diet and citrulline $1 \mathrm{~g} / \mathrm{kg}$ per $\mathrm{d}$ (WDC; $n$ 10)

(Mean values and standard deviations)

\begin{tabular}{|c|c|c|c|c|c|c|c|c|}
\hline & \multicolumn{2}{|c|}{$C(n 10)$} & \multicolumn{2}{|c|}{ WD $(n$ 13) } & \multicolumn{2}{|c|}{ WDA $(n$ 10) } & \multicolumn{2}{|c|}{$\operatorname{WDC}(n 10)$} \\
\hline & Mean & SD & Mean & SD & Mean & SD & Mean & SD \\
\hline$B W(g) \dagger$ & $500 \cdot 1$ & $47 \cdot 7$ & $529 \cdot 6$ & 70.5 & $531 \cdot 1$ & $52 \cdot 6$ & $550 \cdot 0$ & $72 \cdot 8$ \\
\hline Visceral fat $(\mathrm{g}) \dagger$ & $20 \cdot 1$ & $7 \cdot 3$ & $36 \cdot 1^{*}$ & $18 \cdot 0$ & $36 \cdot 6^{\star}$ & 11.7 & 30.8 & 6.4 \\
\hline Skin + cutaneous fat $(\mathrm{g}) \dagger$ & $101 \cdot 8$ & $15 \cdot 5$ & $118 \cdot 6$ & 23.7 & 121.6 & 19.9 & $116 \cdot 9$ & $19 \cdot 0$ \\
\hline Lean mass $(\%$ BW $) \dagger$ & 63.5 & $2 \cdot 8$ & $59 \cdot 1^{\star}$ & $4 \cdot 3$ & $58 \cdot 4^{\star}$ & 3.5 & $60 \cdot 4^{*}$ & 2.4 \\
\hline Food intake $(\mathrm{g} / \mathrm{d}) \ddagger$ & 30.5 & $3 \cdot 3$ & $17 \cdot 4^{\star}$ & 3.9 & $18 \cdot 6^{*}$ & 3.2 & $18 \cdot 3^{*}$ & $2 \cdot 0$ \\
\hline Carbohydrate $(\mathrm{g}) \ddagger$ & $18 \cdot 3$ & $2 \cdot 0$ & $6 \cdot 1^{\star}$ & 1.4 & $6 \cdot 5^{\star}$ & 1.1 & $6 \cdot 4^{*}$ & 0.7 \\
\hline Protein $(\mathrm{g}) \ddagger$ & 4.9 & 0.5 & $3 \cdot 5^{\star}$ & 0.8 & $3 \cdot 7^{\star}$ & 0.6 & $3.7^{\star}$ & 0.4 \\
\hline Lipid $(\mathrm{g}) \ddagger$ & 0.9 & 0.1 & $7 \cdot 8^{\star}$ & 1.8 & $8.4^{*}$ & 1.5 & $8 \cdot 2^{*}$ & 0.9 \\
\hline Fructose $(\mathrm{g}) \ddagger$ & 0 & & $7 \cdot 7$ & $2 \cdot 2$ & 7.5 & $2 \cdot 2$ & $7 \cdot 7$ & $2 \cdot 2$ \\
\hline Energy intake $(\mathrm{kJ} / \mathrm{d})^{*}$ & $421 \cdot 7$ & $45 \cdot 2$ & $459 \cdot 1$ & 74.0 & 478.2 & 61.5 & $476 \cdot 1$ & $37 \cdot 6$ \\
\hline Energy intake $(\mathrm{kcal} / \mathrm{d}) \ddagger$ & $100 \cdot 8$ & $10 \cdot 8$ & $109 \cdot 7$ & $17 \cdot 7$ & 114.3 & 14.7 & 113.8 & 9.0 \\
\hline $\mathrm{N}$ balance $(\mathrm{mg} / 24 \mathrm{~h}) \dagger$ & 344.7 & 144.5 & 366.8 & 80.1 & 372.5 & 76.9 & 226.5 & 191.8 \\
\hline
\end{tabular}

BW, body weight.

* Mean value was significantly different from that of the control group $(P<0.05)$.

$\dagger$ Body weight, body composition and $\mathrm{N}$ balance at 8 weeks.

$\ddagger$ Mean daily intake

Table 2. Effects of citrulline and non-essential amino acid supplementation on metabolic parameters in rats fed a control diet (C; $n$ 10), a Western diet (WD; $n 13$ ), a Western diet and non-essential amino acids (WDA; $n$ 10) or a Western diet and citrulline $1 \mathrm{~g} / \mathrm{kg}$ per d (WDC; $n$ 10)

(Mean values and standard deviations)

\begin{tabular}{|c|c|c|c|c|c|c|c|c|}
\hline & \multicolumn{2}{|c|}{$\mathrm{C}(n 10)$} & \multicolumn{2}{|c|}{$\mathrm{WD}(n 13)$} & \multicolumn{2}{|c|}{ WDA $(n 10)$} & \multicolumn{2}{|c|}{ WDC $(n 10)$} \\
\hline & Mean & SD & Mean & SD & Mean & SD & Mean & SD \\
\hline TAG $(\mathrm{mmol} / \mathrm{l})$ & 0.6 & 0.2 & $1.9^{\star}$ & 1.4 & 1.3 & 0.6 & $1.4^{\star}$ & 0.8 \\
\hline Cholesterol $(\mathrm{mmol} / \mathrm{l})$ & $1 \cdot 1$ & 0.2 & $1.8^{\star}$ & 0.7 & $1 \cdot 8^{*}$ & 0.6 & 1.6 & 0.3 \\
\hline Insulin $(\mu \mathrm{g} / \mathrm{l})$ & 0.6 & 0.2 & $2 \cdot 7^{\star}$ & 2.6 & $1 \cdot 1 \dagger$ & 0.7 & $1.4 \dagger$ & 0.7 \\
\hline Glucose (mmol/l) & 11.4 & 3.0 & $18 \cdot 2^{*}$ & 3.1 & $21 \cdot 4^{*} \dagger$ & 3.5 & $20 \cdot 6^{\star}$ & 4.7 \\
\hline HOMA-IR & 8.0 & $2 \cdot 3$ & $53.9^{*}$ & 53.6 & $24.7 \dagger$ & 16.9 & 32.4 & $21 \cdot 2$ \\
\hline Uric acid $(\mu \mathrm{mol} / \mathrm{l})$ & 23.4 & 7.6 & $27 \cdot 7$ & 20.0 & 19.9 & 5.5 & 23.0 & $10 \cdot 1$ \\
\hline Urea $(\mathrm{mmol} / \mathrm{l})$ & $5 \cdot 2$ & 1.1 & $3.9^{*}$ & 1.2 & $4 \cdot 1^{*}$ & 0.4 & $4 \cdot 1^{\star}$ & 1.0 \\
\hline
\end{tabular}

HOMA-IR, homoeostatic model assessment - insulin resistance.

* Mean value was significantly different from that of the control group $(P<0.05)$

† Mean value was significantly different from that of the WD group $(P<0.05)$

in lean mass tended to be lower compared with the $\mathrm{C}$ group. Cutaneous fat mass and $\mathrm{N}$ balance were not modified by the Western diet.

\section{Effect of diet on metabolic parameters}

Western diet-fed rats had higher plasma TAG $(P<0.05 \mathrm{WD})$, cholesterol $(P<0.01)$, insulin $(P<0.05)$ and glucose $(P<0.001)$ than the control rats (Table 2). NEAA or Cit supplementations lowered plasma TAG by 39 and $32 \%$, respectively, and plasma insulin $(P<0.05)$ compared with the WD group. Only the WD group developed IR as shown by the significantly higher HOMA-IR $(P<0.05)$ compared with the control group. Insulin sensitivity was not different from the control group in WDA and WDC groups; it was significantly improved $(P<0.05)$ in the WDA group and tended to improve $(P=0 \cdot 1)$ in the WDC group compared with the WD group (Table 2). Plasma uric acid did not differ among the groups (Table 2).
Western diet intake resulted in decreased plasma urea $(P<0.05)$, and this was not modified by NEAA or Cit (Table 2). In parallel, the plasma AA profile was modified by the Western diet (Table 3) with a significant increase $(P<0.05)$ in plasma glutamine $(\mathrm{Gln})$, threonine, alanine, proline and lysine (Lys), and a significant decrease $(P<0.05)$ in plasma glycine and isoleucine. Supplementation of the Western diet with NEAA or Cit prevented the modifications in threonine and proline levels $(P<0.05)$, whereas only Cit prevented the modifications in Gln levels. Arg plasma concentration was $14 \%$ higher in the WDC group than in the WD group. In parallel, the ratio of Arg:(ornithine (Orn) + Cit), which can be considered as a marker of systemic Arg bioavailability $^{(25)}$, was higher with Cit than in the WD group, but this did not reach significance (Fig. 1(a)). Moreover, we observed a significant increase $(P<0.05)$ in plasma Lys in all the Western diet-fed groups, and the Arg:Lys ratio, which is an important factor for Arg entry into cells ${ }^{(26)}$, 
Table 3. Effects of citrulline and non-essential amino acid supplementation on plasma amino acids in rats fed a control diet (C; $n$ 10), a Western diet (WD; $n$ 13), a Western diet and non-essential amino acids (WDA; $n 10$ ) or a Western diet and citrulline $1 \mathrm{~g} / \mathrm{kg}$ per $\mathrm{d}$ (WDC; $n$ 10)

(Mean values and standard deviations)

\begin{tabular}{|c|c|c|c|c|c|c|c|c|}
\hline \multirow[b]{2}{*}{ Amino acids $(\mu \mathrm{mol} / \mathrm{l})$} & \multicolumn{2}{|c|}{$\mathrm{C}(n 10)$} & \multicolumn{2}{|c|}{ WD (n 13) } & \multicolumn{2}{|c|}{ WDA $(n 10)$} & \multicolumn{2}{|c|}{ WDC $(n 10)$} \\
\hline & Mean & $\mathrm{SD}$ & Mean & SD & Mean & SD & Mean & $\mathrm{SD}$ \\
\hline Cit & 74 & 13 & 79 & 56 & 68 & 15 & 72 & 14 \\
\hline Gln & 706 & 100 & $805^{\star}$ & 96 & 755 & 74 & $702 \uparrow$ & 81 \\
\hline Arg & 149 & 17 & 144 & 33 & 149 & 16 & 168 & 26 \\
\hline Orn & 71 & 15 & 72 & 17 & 68 & 15 & 62 & 11 \\
\hline Tau & 213 & 36 & 225 & 30 & 232 & 48 & 213 & 34 \\
\hline Met & 58 & 6 & 58 & 9 & 56 & 7 & 59 & 9 \\
\hline Cys & 67 & 15 & 63 & 10 & 73 & 11 & 70 & 10 \\
\hline Asp & 11 & 3 & 16 & 5 & 15 & 7 & 14 & 5 \\
\hline Thr & 320 & 40 & $400^{*}$ & 108 & $340 \dagger$ & 43 & $324 \dagger$ & 45 \\
\hline Ser & 353 & 49 & 377 & 59 & 360 & 28 & 354 & 45 \\
\hline Asn & 86 & 12 & 104 & 17 & 101 & 9 & 96 & 15 \\
\hline Ala & 511 & 76 & $859^{*}$ & 155 & $798^{*}$ & 265 & $727^{*}$ & 139 \\
\hline Pro & 152 & 16 & $253^{\star}$ & 115 & 173† & 17 & 173† & 20 \\
\hline Gly & 376 & 92 & $218^{*}$ & 67 & $236^{*}$ & 49 & $196^{*}$ & 44 \\
\hline Leu & 211 & 39 & 179 & 24 & 181 & 31 & 175 & 32 \\
\hline Ile & 141 & 26 & $103^{*}$ & 19 & $106^{*}$ & 17 & $102^{*}$ & 21 \\
\hline Val & 267 & 43 & 239 & 50 & $224^{*}$ & 34 & $211^{\star}$ & 29 \\
\hline Lys & 394 & 67 & $529^{*}$ & 123 & $465^{\star}$ & 58 & $466^{\star}$ & 78 \\
\hline Phe & 75 & 12 & 72 & 6 & 74 & 7 & 76 & 10 \\
\hline Tyr & 103 & 22 & 103 & 17 & 89 & 8 & 101 & 14 \\
\hline Glu/(Ser + Gly) & 0.12 & 0.06 & $0 \cdot 20^{*}$ & 0.09 & $0.17^{*}$ & 0.04 & $0.18^{\star}$ & 0.04 \\
\hline
\end{tabular}

* Mean value was significantly different from that of the control group $(P<0.05)$.

$\dagger$ Mean value was significantly different from that of the WD group $(P<0.05)$.

(a)

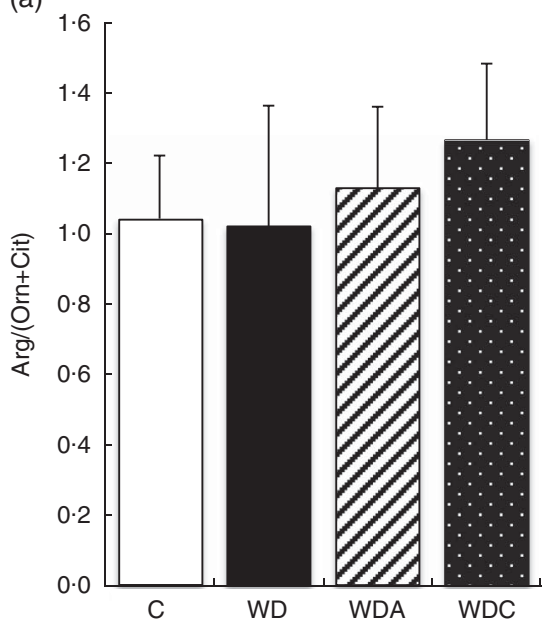

(b)

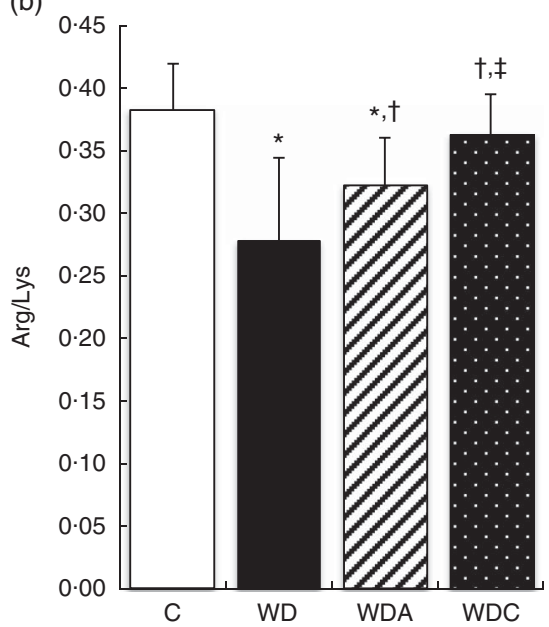

Fig. 1. Effect of amino acids on arginine homoeostasis in rats fed a control diet (C; $n$ 10), a Western diet (WD; $n$ 13), a Western diet and non-essential amino acids (WDA; $n$ 10) or a Western diet and citrulline $1 \mathrm{~g} / \mathrm{kg}$ per d (WDC; $n$ 10). (a) Plasma Arg:(Orn + Cit) ratio, a marker of Arg metabolic utilisation. (b) Plasma Arg:Lys ratio, a marker of Arg bioavailability. Values are means, with standard deviations represented by vertical bars. Mean values were significantly different $(P<0.05)$ * $v$. $C$, $\dagger v$. WD, or $\ddagger v$. WDA.

was decreased in WD $(P=0.0005)$ and WDA $(P=0.002)$ groups (Fig. 1(b)). Only in the Cit-supplemented group this ratio was not different compared with controls $(P=0 \cdot 2)$ and was significantly increased compared with WDA $(P=0.01)$. Finally, we observed an increase in glutathione turnover, characterised by the ratio of glutamate:(serine + glycine) ${ }^{(27)}$ (Table 3 ) in all the Western diet-fed groups $(P<0.05)$.

\section{Effects of non-essential amino acids and citrulline on Western diet-induced hepatic steatosis}

Chronic consumption of Western diet resulted in a significant increase $(P<0.05)$ in liver weight (Fig. 2(a)), and this was associated with a significant increase in hepatic TAG content (Fig. 2(b)) in the WD $(P=0.004)$ and WDA $(P=0 \cdot 02)$ groups. Hepatic TAG content did not differ in the WDC group from the 
(a)

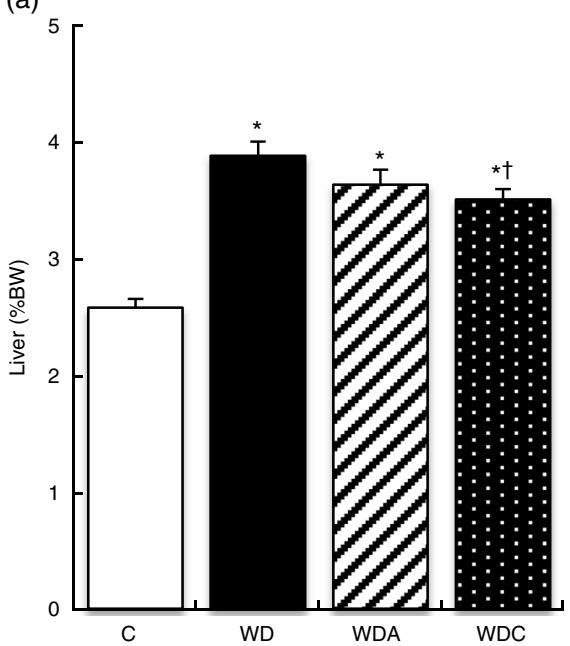

(b)

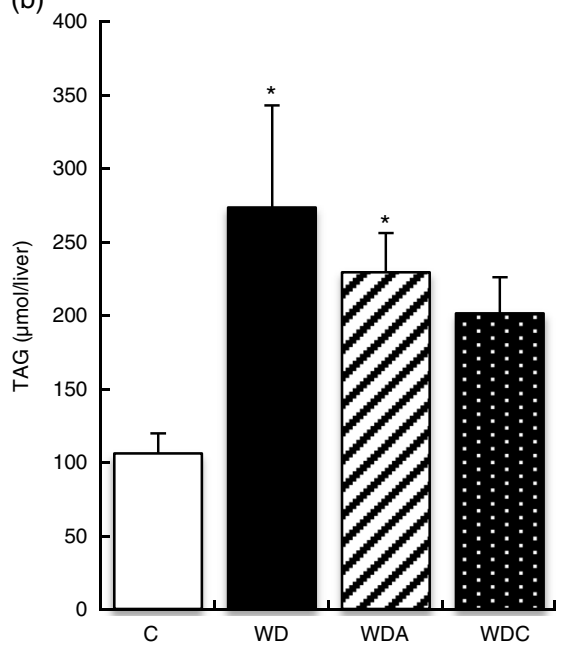

(c)
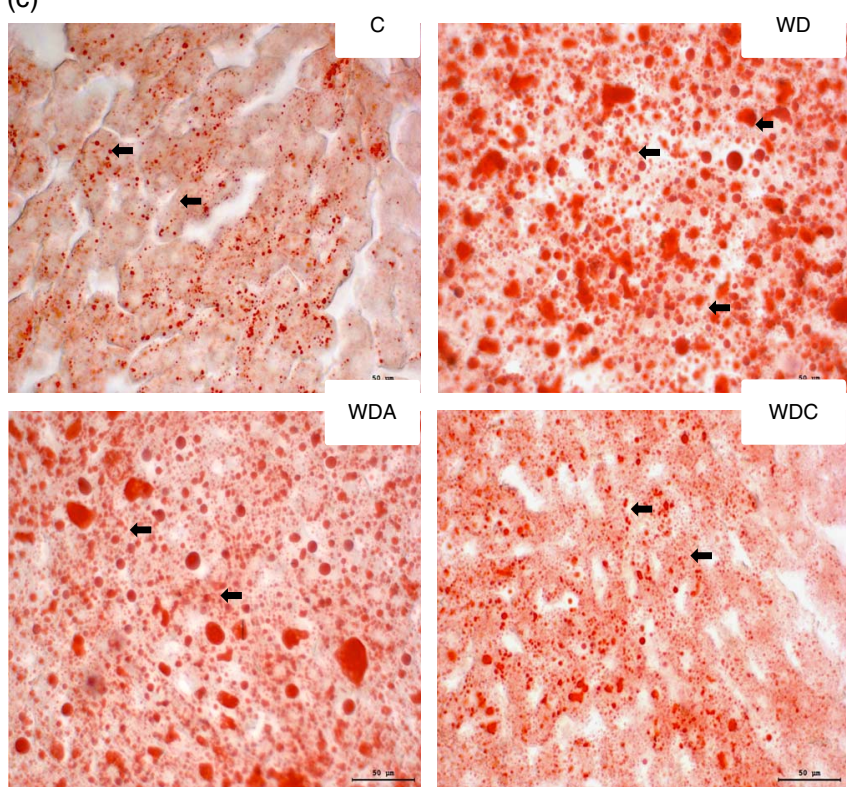

(d)

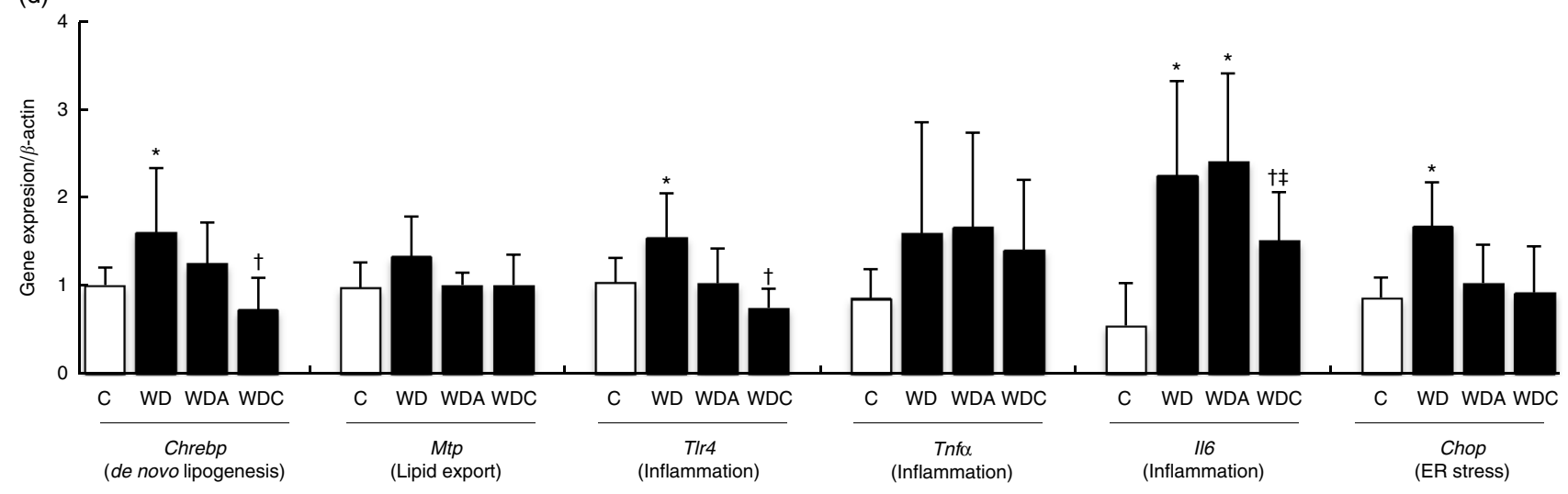

Fig. 2. Effect of amino acids on hepatic lipid accumulation in rats fed a control diet (C; $n$ 10), a Western diet (WD; $n$ 13), a Western diet and non-essential amino acids (WDA; $n$ 10) or a Western diet and citrulline $1 \mathrm{~g} / \mathrm{kg}$ per d (WDC; $n$ 10). (a) Liver weight. (b) Hepatic TAG content. (c) Representative photomicrographs of liver sections stained with Oil Red O showing microvesicular and macrovesicular lipid droplets. $\longrightarrow$, Micro- and macro-vesicular lipid droplets. (d) Expression of genes involved in hepatic lipid accumulation. Values are means, with standard deviations represented by vertical bars. Mean values were significantly different $(P<0.05):{ }^{*} v . C, \dagger v$. WD or $\ddagger v$. WDA. 
Table 4. Effects of citrulline and non-essential amino acid supplementation on the gut-liver axis in rats fed a control diet (C; $n$ 10), a Western diet (WD; $n$ 13), a Western diet and non-essential amino acids (WDA; $n$ 10) or a Western diet and citrulline $1 \mathrm{~g} / \mathrm{kg}$ per $\mathrm{d}$ (WDC; $n$ 10)

(Mean values and standard deviations)

\begin{tabular}{|c|c|c|c|c|c|c|c|c|}
\hline & \multicolumn{2}{|c|}{$C(n 10)$} & \multicolumn{2}{|c|}{ WD (n 13) } & \multicolumn{2}{|c|}{ WDA $(n 10)$} & \multicolumn{2}{|c|}{ WDC $(n 10)$} \\
\hline & Mean & SD & Mean & SD & Mean & SD & Mean & SD \\
\hline \multicolumn{9}{|l|}{ Gut function } \\
\hline Endotoxin (EU/ml) & 0.4 & 0.4 & 0.6 & 0.6 & 0.8 & 0.8 & 1.0 & 0.8 \\
\hline MPO activity (U/mg) & 0.2 & 0.2 & 0.4 & 0.2 & 0.4 & 0.3 & $0.2 \ddagger$ & 0.1 \\
\hline Claudin-1§ & 0.7 & 0.2 & 0.4 & 0.3 & 0.6 & 0.3 & $0.8+$ & 0.1 \\
\hline ZO1§ & 0.6 & 0.2 & 0.4 & 0.3 & 0.6 & 0.4 & 0.4 & 0.2 \\
\hline \multicolumn{9}{|l|}{ Liver function } \\
\hline AST (UI/I) & $71 \cdot 1$ & $9 \cdot 3$ & $68 \cdot 2$ & $15 \cdot 1$ & $66 \cdot 4$ & $21 \cdot 7$ & $65 \cdot 1$ & $12 \cdot 8$ \\
\hline ALT (UI/I) & 34.4 & 4.5 & 38.3 & $16 \cdot 8$ & 34.4 & $17 \cdot 7$ & 29.5 & 11.5 \\
\hline ALP (UI/I) & $95 \cdot 3$ & $20 \cdot 2$ & $124 \cdot 1$ & $33 \cdot 2$ & $99 \cdot 1$ & $15 \cdot 4$ & 93.7 & 33.6 \\
\hline Bilirubin $(\mu \mathrm{mol} / \mathrm{l})$ & 1.0 & 0.0 & 1.4 & 0.5 & $1 \cdot 1$ & 0.3 & 1.2 & 0.4 \\
\hline GSSG/GSH & $6 \cdot 5$ & $2 \cdot 2$ & $3 \cdot 5^{\star}$ & 0.8 & $3 \cdot 8^{*}$ & 1.4 & $4 \cdot 7^{\star}$ & 1.5 \\
\hline
\end{tabular}

MPO, myeloperoxydase; ZO1, zonula occludens 1; AST, aspartate aminotransferase; ALT, alanine aminotransferase; ALP, alkaline phosphatase.

* Mean value was significantly different from that of the control group $(P<0.05)$

$\dagger$ Mean value was significantly different from that of the WD group $(P<0.05)$.

$\ddagger$ Mean value was significantly different from that of the WDA group $(P<0.05)$.

$\S$ Normalised to glyceraldehyde 3-phosphate dehydrogenase.

C group, and liver weight was significantly lower $(P<0.05)$ in the WDC group compared with the WD group (Fig. 2(a)). In parallel, plasma bilirubin, ALP, ALT and AST levels were not modified by the different diets (Table 4).

On liver histological examination, WD and WDA rats presented macrovesicular and microvesicular lipid droplets, whereas in Cit-supplemented animals hepatic lipids were limited to microvesicular lipid droplets (Fig. 2(c)).

Hepatic steatosis in Western diet-fed rats was associated with an increased expression of Chrebp $(P<0.05)$ and a trend towards increased expression of Mtp (Fig. 2(d)). Only Cit administration abolished WD-induced increase in Chrebp expression.

Western diet feeding was associated with liver inflammation, as suggested by the induction of Tlr4 expression in the WD group $(P<0.05)$ and $I l 6$ expression in the WD and WDA groups $(P<0.05)$, as well as the slight increase in Tnf $\alpha$ (Fig. 2(d)). Only Cit administration significantly decreased $(P<0.05)$ Tlr 4 and $I l 6$ mRNA expressions.

The Western diet significantly decreased $(P<0.05)$ the GSSG: GSH ratio, a marker of oxidative stress, without any effect of NEAA, whereas the ratio tended to increase $(P=0.09 v$. WD) with Cit supplementation (Table 4).

The Western diet also led to ER stress, as shown by the significant increase $(P<0.05)$ in Chop expression, and it was prevented by NEAA and Cit supplementation (Fig. 3(c)).

\section{Effect of diet on gut microbiota}

We did not observe any modification in total caecal microbiota or Bifidobacteria count. Compared with the control diet, the main effect of the Western diet on caecal microbiota was a marked increase in A. muciniphila (online Supplementary Fig. S1). NEAA and Cit did not modify Western diet-induced changes in caecal microbiota.
In contrast, the Western diet induced dramatic changes in the colon mucosa-associated microbiota, with a significant decrease $(P<0.05)$ in total bacteria, C. leptum, Bacteroides/Prevotella and Lactobacillus/Leuconostoc (Fig. 3(a)-(h)). Colonisation with A. muciniphila was lower in the WD group than in the controls (Fig. 3(h)), but this did not reach significance. Although NEAA or Cit failed to significantly counterbalance the effect of the Western diet on total bacteria and C. leptum, only Cit restored Bacteroides/Prevotella colonisation to control levels, but only at the mucosal level.

\section{Effect of diet on gut function (Table 4)}

At gut level, the Western diet did not increase plasma endotoxin levels.

MPO activity slightly increased in colon mucosa in Western diet-fed rats compared with the controls. It was significantly lower in the WDC group compared with the WDA group $(P=0.03)$.

Expression of the tight-junction protein claudin- 1 in the colon tended to be decreased by the Western diet $(P=0 \cdot 06)$, and this was associated with increased Tnfo and Tlr4 expressions $(P<0.05)$ (Fig. 4(a)). NEAA and Cit administration significantly decreased Tlr4 expression $(P<0 \cdot 05)$, whereas Cit decreased Tnfo expression $(P<0.05)$, and tended to increase claudin-1 protein expression (ANOVA $P=0 \cdot 1$, WD $v$. WDC $P=0 \cdot 02$ ). Cytoplasmic protein expression of ZO-1 was preserved among groups.

\section{Discussion}

The progression of non-alcoholic fatty liver (NAFL) to its severe necro-inflammatory stage - that is, NASH - may be prevented through the management of lipid metabolism and inflammatory processes. The benefits of AA supply on the development of 
(a)

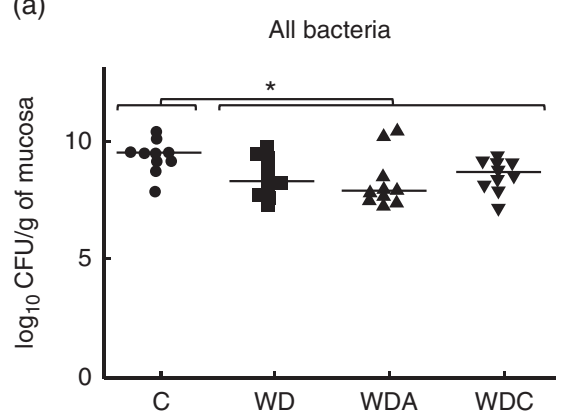

(c)

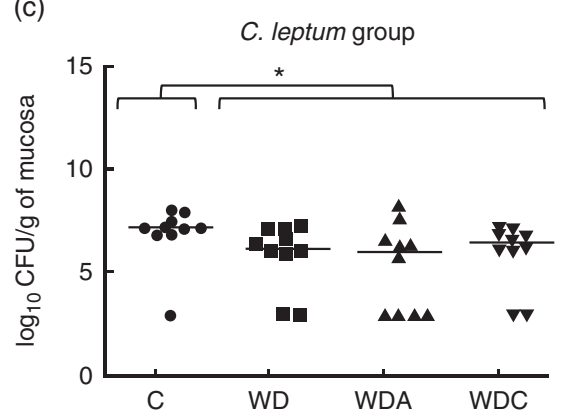

(e)

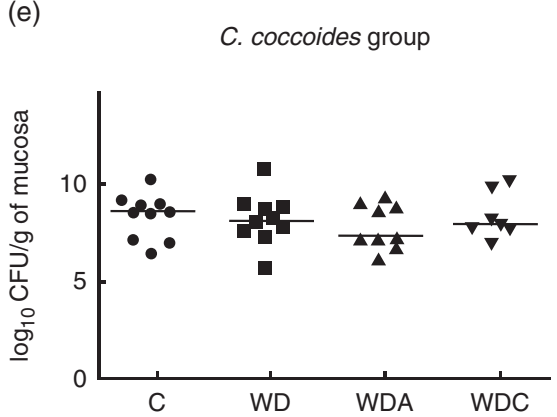

(g)

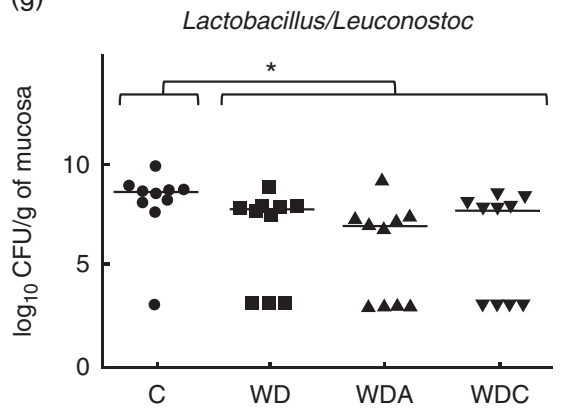

(b)

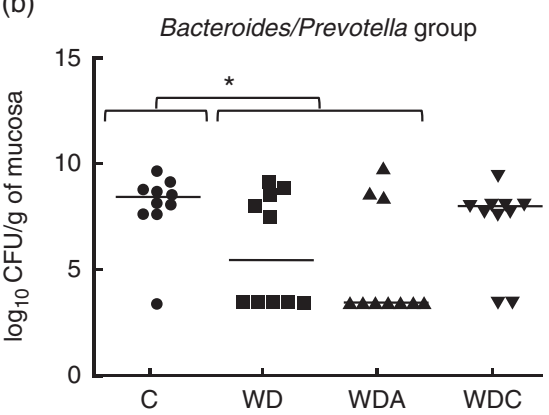

(d)

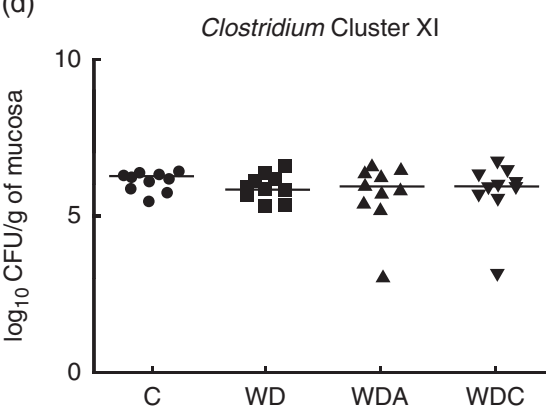

(f)

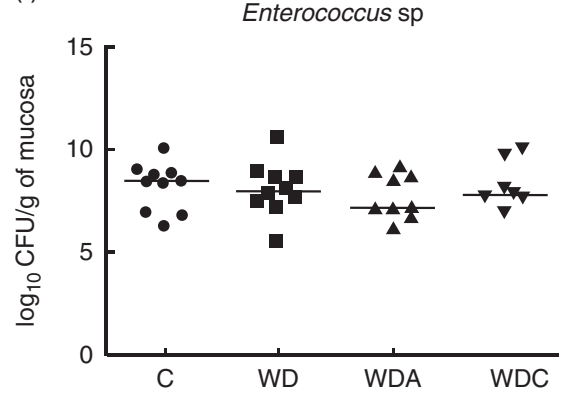

(h)

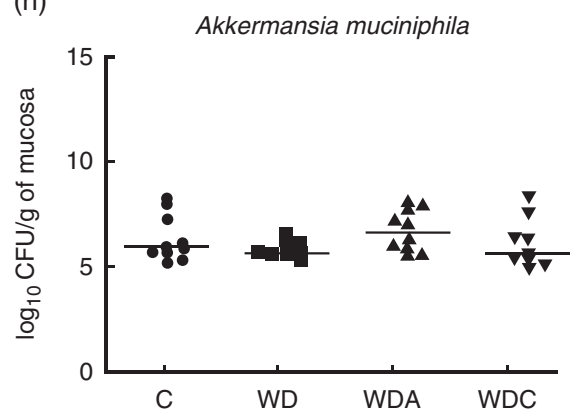

Fig. 3. Effect of amino acids on microbiota in rats fed a control diet (C; $n$ 10), a Western diet (WD; $n$ 13), a Western diet and non-essential amino acids (WDA; $n$ 10) or a Western diet and citrulline $1 \mathrm{~g} / \mathrm{kg}$ per d (WDC; $n$ 10). Quantification of all bacteria populations (a), Bacteroides/Prevotella group (b), Clostridium leptum group (c), Cluster XI (d), Coccoides group (e), enterococcus (f), Lactobacillus/Leuconostoc group (g) and Akkermansia muciniphila (h) in the colon mucosa in all groups. $\mathrm{CFU}$, colony-forming units. - Rat; $\_$, median. * Mean values were significantly different compared to that in $\mathrm{C}$ group $(P<0.05)$.

NAFL have been repeatedly demonstrated, and we have previously shown a preventive effect of Cit on fructose-induced NAFL. In the present study, we evaluated whether Cit may also be effective during Western diet feeding, which is known to affect gut function and NAFLD progression. Our present results showed that in a situation of chronic Western diet feeding, Cit supplementation presents some specific beneficial effects on several factors of disease progression and this could probably decrease the severity of steatosis.

First, as expected, the Western diet induced hepatic steatosis, as shown by increased liver weight and TAG content, via increased Chrebp expression and the induction of de novo lipogenesis. Liver histological examination showed macrovesicular and microvesicular lipid droplet accumulation in the 
(a)

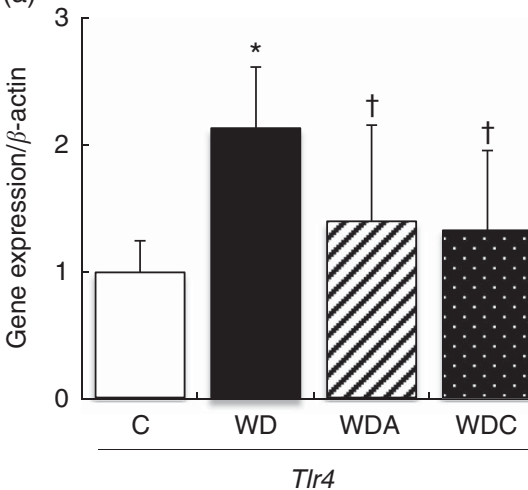

(b)
WD group, suggesting progression of the disease ${ }^{(28)}$. Only in the Cit-supplemented group, a lower TAG content was observed with lipid accumulation limited to microvesicular lipid droplets, and this was probably related to the lower expression of Chrebp, a key regulator of de novo lipogenesis. As a consequence, Cit significantly decreased liver weight compared with the WD group. The effect of Cit on lipid metabolism may be the result of decreased de novo lipogenesis, but also to a better VLDL-TAG release given the improved peripheral TAG oxidation previously demonstrated ${ }^{(29)}$.

Chronic Western diet consumption in our experiments did not induce weight gain. Owing to the higher energy density of their diet and to fructose in their drinking water, the Western diet-fed rats had a energy intake similar to control rats, despite significantly decreased food intake, probably related to the satiety effect of fructose $^{(30)}$. In the literature, WD-induced increase in BW is only observed with a higher energy intake ${ }^{(31,32)}$.

NAFLD is commonly associated with higher adiposity. In this study, the Western diet increased visceral fat mass, and this was dampened by Cit supplementation in keeping with several studies from our group ${ }^{(18,33)}$. The effect of Cit may occur through enhanced visceral adipose tissue lipolysis ${ }^{(34,35)}$, fat oxidation $^{(34)}$ and uncoupling of the respiratory chain, resulting in energy expenditure that favours fat mass reduction ${ }^{(36)}$. It will be of interest to evaluate whether Cit may modify the fatty acid profile in adipose tissue, and thereby regulate fat cell size. Indeed, Garaulet et $a l .{ }^{(37)}$ showed that fatty acid composition of adipose tissue is correlated to adipocyte size and number in overweight/obese humans. An additional mechanism may also be involved. Indeed Vasanthakumar et al. ${ }^{(38)}$ showed that an increase in visceral fat mass may also be associated with a reduction in regulatory $\mathrm{T}$ cells, which prevents obesityassociated inflammation and preserves insulin sensitivity and glucose tolerance. As Cit is able to limit the polarisation of macrophages to the M1 phenotype in the peritoneal tissues ${ }^{(39)}$, the influence of Cit on regulatory $\mathrm{T}$ cells deserves to be studied.

Western diet-induced visceral adiposity was associated with hepatic and peripheral IR as shown by the significant increase in HOMA-IR, in agreement with literature data ${ }^{(40)}$.
Indeed, dysfunctional adipose tissue, especially the more metabolically active visceral fat tissue, plays a central role in the pathogenesis of NASH and IR through the release of cytokines and fatty acids ${ }^{(40,41)}$. In our experiments, NEAA and Cit corrected the higher plasma insulin level and improved insulin sensitivity. In addition to its action on body fat distribution, Cit may act on IR via its effects on Arg availability and nitric oxide synthesis, which plays an important role in the regulation of energy metabolism ${ }^{(42)}$. Although we have not investigated nitric oxide production, Cit did prevent Western diet-induced alterations in Arg metabolism, as shown by the increase in plasma Arg:Lys and Arg:(Orn + Cit) ratios $^{(25,26)}$. Interestingly, Cit-supplemented rats did not present a higher plasma concentration of this AA compared with non-supplemented rats, suggesting its rapid utilisation in a situation of metabolic disorder

Western diet feeding was also associated with a decrease in lean body mass. Although the mechanisms remain to be investigated, hepatic fat accumulation may alter the liver-muscle axis - for example, through the production of lipids ${ }^{(43)}$, methylglyoxal ${ }^{(44)}$, hepatokines such as Fetuin $\mathrm{A}^{(45)}$ and uric $\operatorname{acid}^{(46)}$ by the liver. All these metabolites and lipid infiltration in myocytes decrease insulin sensitivity in muscle, and may contribute to muscle anabolic resistance and loss in lean body mass ${ }^{(43)}$. The preventive effect of Cit on hepatic lipid accumulation probably occurs through its beneficial effect on visceral fat mass, but also at least in part through an activation of muscle protein synthesis ${ }^{(47)}$, taking into account the role of muscle mass on metabolic homoeostasis. Of note, the Western diet-induced loss in lean body mass in our experiments was associated with modifications in the plasma levels of several AA, suggesting disturbances in AA inter-organ exchanges. The decrease in plasma urea in the absence of modifications in $\mathrm{N}$ balance suggests preserved urinary $\mathrm{N}$ elimination and increased renal ammonia excretion. This might be related to a lower gluconeogenic flux from AA, elevated plasma glucose resulting from gluconeogenic fructose utilisation and probably explains the increase in the plasma levels of some gluconeogenic AA. These data are in contrast with our previous 
observation in fructose-induced NAFLD, where Cit corrected fructose-induced perturbations in plasma AA. This is probably related to the high fat content of the diet and requires to be further investigated.

Interestingly, plasma Gln was significantly increased in the WD group and normalised by Cit supply. The increase in Gln may be related to (i) its decreased utilisation in liver ureagenesis and/or (ii) its lower utilisation by the gut. Although IR is associated with increased plasma Cit, plasma Cit was not altered by the Western diet in our experiments. As plasma Cit depends on its intestinal production in part from Gln, this may be related to the deterioration in intestinal function discussed below. Only Cit normalised plasma Gln levels; this may due to its protective effects on the gut leading to improved Gln intestinal utilisation.

Liver inflammation is a hallmark of NASH, and is associated with increased production of pro-inflammatory cytokines such as TNF $\alpha$ or IL6. Increased plasma cytokine levels may originate from the liver and the gut but also from dysfunctional adipose tissue as mentioned above or other immune organs such as the spleen $^{(48)}$. The exact role of IL6 remains to be investigated, as some studies have clearly demonstrated that IL6 plays a key role in determining IR and other NAFLD-related diseases ${ }^{(48)}$, whereas other studies have shown an hepatoprotective effect of IL6 ${ }^{(49)}$. Focusing on the liver, hepatic lipid overload increases macrophage recruitment leading to a higher production of cytokines such as TNF $\alpha$ and IL6. Our present study shows that the Western diet increased hepatic Tlr4 and Il6 mRNA expressions, suggesting liver inflammation, and this is prevented by Cit supplementation. Increase in Tlr4 expression may also reflect an increase in macrophage infiltration in the liver ${ }^{(50)}$. The Western diet could induce a hepatic macrophage switch to an M1 phenotype, and Cit could promote an M2 phenotype as shown by the lower ILG mRNA expression. Further studies are needed to investigate this point. Interestingly, the Western diet was associated with higher hepatic expression of the pro-apoptotic Chop gene, suggesting endoplasmic reticulum stress, which was significantly reduced by NEAA and Cit. Whatever the mechanism involved, our data showed that Cit prevented Western diet-induced hepatic inflammation. In addition, oxidative stress could be both a cause and a consequence of the progression of NAFLD to NASH. It can mediate liver injury through at least two major pathways: direct cell injury and cell signalling. Hepatic lipid accumulation and IR can produce endoplasmic reticulum stress, leading to activation of NF- $\kappa \mathrm{B}$, which plays a critical role in the production of pro-inflammatory cytokines. This study showed that the Western diet increased glutathione turnover, suggesting an induction of antioxidant defences, and decreased hepatic GSSG:GSH ratio, a marker of oxidative stress. Cit tended to normalise GSSG:GSH ratio, whereas NEAA were ineffective.

Alterations in gut permeability and microbiota play a critical role in disease progression ${ }^{51,52)}$. The Western diet has been shown to be associated with reduced bacterial colonisation, but increased numbers of mucin-degrading bacteria of the Bacteroidetes and Lachnospiraceae family/Firmicutes phyla ${ }^{(53)}$. In our study, an increase in mucin-degrading bacteria was observed in the caecal contents of the Western diet-fed rats without modification in total bacteria, whereas we observed reduced mucosal colonisation. An increase in the Firmicutes: Bacteroidetes ratio has been repeatedly demonstrated to develop with excess weight ${ }^{(54)}$ and in NAFLD as a result of decreased Bacteroidetes ${ }^{(55)}$ or an increase in some members of Firmicutes ${ }^{(56)}$. In the mucosa, in our study, despite decreased Lactobacillus/Leuconostoc and C. leptum groups, some other members of Firmicutes (i.e. other Clostridium clusters such as C. coccoides and enterococci) remained at high levels. On the other hand, the Bacteroides/Prevotella group was significantly decreased in the WD group compared with controls. This bacterial group, a major component of the Bacteroidetes phylum, is recognised for its immunomodulatory properties and its role in gut homoeostasis ${ }^{(57)}$. Finally, A. muciniphila level was not affected by the Western diet. As A. muciniphila has been shown to be able to restore the gut mucus layer, and its abundance is inversely correlated with weight gain ${ }^{(58,59)}$, the fact that all our rats exhibited normal weight probably explains this latter observation. In our study, this dysbiosis was associated with inflammation as shown by the higher MPO activity and expression of TNF $\alpha$ and TLR4 in colon mucosa. We also noted a lower expression of the tight-junction protein claudin-1 in the colon of Western-diet fed rats. The increased caecal content and the mucosal preservation of $A$. muciniphila probably help in explaining why we $\operatorname{did}$ not observe any Western diet-induced endotoxaemia.

Although Cit had little impact on total bacteria and C. leptum, it had a positive effect on the Bacteroides/Prevotella count, which was restored to a level similar to the control. Colonisation of the gut by damaging bacteria could thus be limited by Cit in favour of protective bacteria, leading to a lesser colon mucosa inflammation as observed in our Cit-supplemented rats. The resulting prevention of intestinal inflammation could in turn protect gut barrier function. Several mechanisms, either direct or indirect, may contribute to these effects of Cit on microbiota. First, as already demonstrated for Arg, a closely related AA that affects gut microbiota, we cannot rule out a direct effect of oral Cit on the large intestine. Indeed, as the absorption rate of these AA is relatively slow a small part may arrive in the large intestine and be metabolised by the microbiota. In this respect, Bacteroides/Prevotella are known to synthesise Arg from $\mathrm{Cit}^{(60,61)}$ and Arg, at least in part as a precursor for polyamine synthesis, may protect intestinal mucosa and also play a role in cell growth ${ }^{(62)}$. Second, systemic effects of Cit may be involved. As already mentioned, Cit displays trophic and anti-inflammatory properties on the gut and this is further supported by our study. Through these effects, Cit may thus affect the interaction between gut barrier (immune cell modulation, secretory antimicrobial peptide production) and the microbiota. In addition, the improvement of peripheral insulin sensitivity by Cit may also affect gut barrier/microbiota interactions; indeed insulin has been shown to play an important role, for example, in the production of mucin and the integrity of gut mucous layer. As a result, whatever the underlying mechanisms, Cit supplementation was associated with decreased gut inflammation as indicated by lower MPO activity and pro-inflammatory gene expression.

Finally, in terms of specificity of the effects of Cit, some of our data show that our NEAA supplement, isonitrogenous to Cit, 
may in part prevent some of the metabolic effects of the Western diet. Although this suggests a possible effect of extra $\mathrm{N}$ supply, the effect of the NEAA mixture may be debated. Indeed, although in our study, only low dose of each AA was used, a specific effect of one of the six AA cannot be ruled out. For example, one study showed that histidine significantly decreases gene expression and activity of transcription factors SREBP and CHREBP and lipogenic enzymes Fas and HMG-CoA reductase, leading to decreased hepatic TAG content and cholesterol and improved insulin sensitivity ${ }^{(63)}$. In the same way, Glycine may prevent lipid accumulation in the liver and bloodstream ${ }^{(64)}$, preserve muscle mass, stimulate loss of adipose tissue and also exert anti-inflammatory effects ${ }^{(65)}$. Further studies are needed to clarify this point.

In conclusion, beneficial effects of both NEAA and Cit on some aspects of lipid metabolism confirm that AA supply by itself may be protective on NAFLD. In addition, besides a possible N supply-related effect, Cit seems to play an important specific role. Cit showed a direct effect on several factors of disease progression at the liver level such as lipid accumulation, inflammation, oxidative stress and ER stress. Cit supply is also associated with specific effects at the peripheral level on visceral adipose tissue and gut function. Cit supplementation may thus be an interesting nutritional strategy in the prevention of NAFLD progression to NASH.

\section{Acknowledgements}

The authors thank Servane Le Plenier, Radji Ramassamy and Christelle Vicente for expert technical assistance, and acknowledge the imaging platform and animal facility of Paris Descartes University.

This project was supported by an Institut Appert grant and the French National Research Agency (ANR) grant (ANR-11-BSV1-0015, NAFLD-citrulline)

Study concept and design: J.-P. D. B., S. B. and I. B. Acquisition of data, analysis and interpretation of the data: P. J., K. F., E. N., A.-J. W.-D., M.-J. B. Drafting of the manuscript: P. J. and J.-P. D. B. Critical revision of the manuscript for important intellectual content: all authors.

Professor J.-P. D. B. is a shareholder of Citrage Company.

\section{Supplementary material}

For supplementary material/s referred to in this article, please visit http://dx.doi.org/doi:10.1017/S0007114516001793

\section{References}

1. Tarantino G (2007) Should nonalcoholic fatty liver disease be regarded as a hepatic illness only? World J. Gastroenterol 13, 4669-4672.

2. Pinzani M (2011) Pathophysiology of non-alcoholic steatohepatitis and basis for treatment. Dig Dis 29, 243-248.

3. Zhang X-Q, Xu C-F, Yu C-H, et al. (2014) Role of endoplasmic reticulum stress in the pathogenesis of nonalcoholic fatty liver disease. World J Gastroenterol 20, 1768-1776.

4. Takaki A, Kawai D \& Yamamoto K (2014) Molecular mechanisms and new treatment strategies for non-alcoholic steatohepatitis (NASH). Int J Mol Sci 15, 7352-7379.
5. Volynets V, Küper MA, Strahl S, et al. (2012) Nutrition, intestinal permeability, and blood ethanol levels are altered in patients with nonalcoholic fatty liver disease (NAFLD). Dig Dis Sci 57, 1932-1941.

6. Sobrecases H, Lê K-A, Bortolotti M, et al. (2010) Effects of short-term overfeeding with fructose, fat and fructose plus fat on plasma and hepatic lipids in healthy men. Diabetes Metab 36, 244-246.

7. Ilan Y (2012) Leaky gut and the liver: a role for bacterial translocation in nonalcoholic steatohepatitis. World J. Gastroenterol 18, 2609-2618.

8. Chassaing B, Etienne-Mesmin L \& Gewirtz AT (2014) Microbiota-liver axis in hepatic disease. Hepatology 59, 328-339.

9. Thuy S, Ladurner R, Volynets V, et al. (2008) Nonalcoholic fatty liver disease in humans is associated with increased plasma endotoxin and plasminogen activator inhibitor 1 concentrations and with fructose intake. J Nutr 138, $1452-1455$.

10. Chalasani N, Younossi Z, Lavine JE, et al. (2012) The diagnosis and management of non-alcoholic fatty liver disease: practice Guideline by the American Association for the Study of Liver Diseases, American College of Gastroenterology, and the American Gastroenterological Association. Hepatology 55, 2005-2023

11. Theytaz F, Noguchi Y, Egli L, et al. (2012) Effects of supplementation with essential amino acids on intrahepatic lipid concentrations during fructose overfeeding in humans. Am J Clin Nutr 96, 1008-1016.

12. Lee S-M, Han HW \& Yim SY (2014) Beneficial effects of soy milk and fiber on high cholesterol diet-induced alteration of gut microbiota and inflammatory gene expression in rats. Food Funct 6, 492-500.

13. Lopez-Legarrea P, Fuller NR, Zulet MA, et al. (2014) The influence of Mediterranean, carbohydrate and high protein diets on gut microbiota composition in the treatment of obesity and associated inflammatory state. Asia Pac J Clin Nutr 23, 360-368.

14. Rhoads MJ \& Wu G (2009) Glutamine, arginine, and leucine signaling in the intestine. Amino Acids 37, 111-122.

15. Ginguet A, Cynober L \& De Bandt J-P (2016) Indications for infusing individual amino acids (arginine, glutamine, taurine, carnitine, leucine, citrulline) in critical illness? Curr Opin Clin Nutr Metab Care 19, 161-169.

16. Antunes MM, Leocádio PCL, Teixeira LG, et al. (2016) Pretreatment with L-citrulline positively affects the mucosal architecture and permeability of the small intestine in a murine mucositis model. J Parenter Enteral Nutr 40, 279-286.

17. Lai C-H, Lee C-H, Hung C-Y, et al. (2015) Oral citrulline mitigates inflammation and jejunal damage via the inactivation of neuronal nitric oxide synthase and nuclear factor-kb in intestinal ischemia and reperfusion. J Parenter Enteral Nutr (Epublication ahead of print 30 June 2015).

18. Jegatheesan PP, Beutheu S, Ventura G, et al. (2015) Effect of specific amino acids on hepatic lipid metabolism in fructose-induced non-alcoholic fatty liver disease. Clin Nutr 35, 175-182

19. Jegatheesan P, Beutheu S, Ventura G, et al. (2015) Citrulline and nonessential amino acids prevent fructose-induced nonalcoholic fatty liver disease in rats. J. Nutr 145, 2273-2279.

20. Osowska S, Moinard C, Neveux N, et al. (2004) Citrulline increases arginine pools and restores nitrogen balance after massive intestinal resection. Gut 53, 1781-1786.

21. Ren W, Chen S, Yin J, et al. (2014) Dietary arginine supplementation of mice alters the microbial population and activates intestinal innate immunity. J Nutr 144, 988-995. 
22. Barone FC, Hillegass LM, Price WJ, et al. (1991) Polymorphonuclear leukocyte infiltration into cerebral focal ischemic tissue: myeloperoxidase activity assay and histologic verification. J Neurosci Res 29, 336-345.

23. Beutheu Youmba S, Belmonte L, Galas L, et al. (2012) Methotrexate modulates tight junctions through NF-KB, MEK, and JNK pathways. J Pediatr Gastroenterol Nutr 54, 463-470.

24. Kalach N, Kapel N, Waligora-Dupriet A-J, et al. (2013) Intestinal permeability and fecal eosinophil-derived neurotoxin are the best diagnosis tools for digestive nonIgE-mediated cow's milk allergy in toddlers. Clin Chem Lab Med 51, 351-361.

25. Sailer M, Dahlhoff C, Giesbertz P, et al. (2013) Increased plasma citrulline in mice marks diet-induced obesity and may predict the development of the metabolic syndrome. PLOS ONE 8, e63950.

26. Wu G, Bazer FW, Davis TA, et al. (2009) Arginine metabolism and nutrition in growth, health and disease. Amino Acids 37, 153-168.

27. Vanni E, Rosso C, Mezzabotta L, et al. (2014) Metabolomic analysis identifies new biomarkers of liver damage in NAFLD. Hepatology 60, Suppl., 227A.

28. Ibrahim M, Singh C, Ganie MA, et al. (2009) NASH: the hepatic injury of metabolic syndrome: a brief update. Int J Health Sci (Qassim) 3, 265-270.

29. Moussaoui F, Binimbi A, Cottart CH, et al. (2009) Hepatoprotective effect of citrulline against ischemia-reperfusion injury in rat. J Hepatol 50, 568.

30. Moran TH (2009) Fructose and satiety. J Nutr 139, 1253S-1256S.

31. Tsuchiya H, Ebata Y, Sakabe T, et al. (2013) High-fat, highfructose diet induces hepatic iron overload via a hepcidinindependent mechanism prior to the onset of liver steatosis and insulin resistance in mice. Metab Clin Exp 62, 62-69.

32. Zelber-Sagi S, Nitzan-Kaluski D, Goldsmith R, et al. (2007) Long term nutritional intake and the risk for non-alcoholic fatty liver disease (NAFLD): a population based study. J Hepatol $\mathbf{4 7}$, 711-717.

33. Moinard C, Le Plenier S, Noirez P, et al. (2015) Citrulline supplementation induces changes in body composition and limits age-related metabolic changes in healthy male rats. J Nutr 145, 1429-1437.

34. Joffin N, Jaubert A-M, Durant S, et al. (2014) Citrulline induces fatty acid release selectively in visceral adipose tissue from old rats. Mol Nutr Food Res 58, 1765-1775.

35. Joffin N, Jaubert A-M, Durant $\mathrm{S}$, et al. (2014) Citrulline reduces glyceroneogenesis and induces fatty acid release in visceral adipose tissue from overweight rats. Mol Nutr Food Res 58 , 2320-2330.

36. Joffin N, Jaubert A-M, Bamba J, et al. (2015) Acute induction of uncoupling protein 1 by citrulline in cultured explants of white adipose tissue from lean and high-fat-diet-fed rats. Adipocyte 4, 129-134.

37. Garaulet M, Hernandez-Morante JJ, Lujan J, et al. (2006) Relationship between fat cell size and number and fatty acid composition in adipose tissue from different fat depots in overweight/obese humans. Int J Obes (Lond) 30, 899-905.

38. Vasanthakumar A, Moro K, Xin A, et al. (2015) The transcriptional regulators IRF4, BATF and IL-33 orchestrate development and maintenance of adipose tissue-resident regulatory T cells. Nat Immunol 16, 276-285.

39. Breuillard C, Bonhomme S, Couderc R, et al. (2015) In vitro antiinflammatory effects of citrulline on peritoneal macrophages in Zucker diabetic fatty rats. Br J Nutr 113, 120-124.

40. Gaggini M, Morelli M, Buzzigoli E, et al. (2013) Non-alcoholic fatty liver disease (NAFLD) and its connection with insulin resistance, dyslipidemia, atherosclerosis and coronary heart disease. Nutrients 5, 1544-1560.
41. De Moura RF, Ribeiro C, de Oliveira JA, et al. (2009) Metabolic syndrome signs in Wistar rats submitted to different high-fructose ingestion protocols. Br J Nutr 101, 1178-1184.

42. Cynober L, Moinard C \& De Bandt J-P (2010) The 2009 ESPEN Sir David Cuthbertson. Citrulline: a new major signaling molecule or just another player in the pharmaconutrition game? Clin Nutr 29, 545-551.

43. Stephens FB, Chee C, Wall BT, et al. (2015) Lipid-induced insulin resistance is associated with an impaired skeletal muscle protein synthetic response to amino acid ingestion in healthy young men. Diabetes 64, 1615-1620.

44. Dhar I, Dhar A, Wu L, et al. (2013) Increased methylglyoxal formation with upregulation of renin angiotensin system in fructose fed Sprague Dawley rats. PLOS ONE 8, e74212.

45. Srinivas PR, Wagner AS, Reddy LV, et al. (1993) Serum alpha 2-HS-glycoprotein is an inhibitor of the human insulin receptor at the tyrosine kinase level. Mol Endocrinol 7 , $1445-1455$.

46. Fabbrini E, Serafini M, Colic Baric I, et al. (2014) Effect of plasma uric acid on antioxidant capacity, oxidative stress, and insulin sensitivity in obese subjects. Diabetes $\mathbf{6 3}$, 976-981.

47. Osowska S, Duchemann T, Walrand S, et al. (2006) Citrulline modulates muscle protein metabolism in old malnourished rats. Am J Physiol Endocrinol Metab 291, E582-E586.

48. Tarantino G, Savastano S, Capone D, et al. (2011) Spleen: a new role for an old player? World J Gastroenterol 17, $3776-3784$.

49. Gao B (2004) Therapeutic potential of interleukin-6 in preventing obesity- and alcohol-associated fatty liver transplant failure. Alcohol 34, 59-65.

50. Bian Z, Peng Y, You Z, et al. (2013) CCN1 expression in hepatocytes contributes to macrophage infiltration in nonalcoholic fatty liver disease in mice. J Lipid Res 54, 44-54.

51. Ritze Y, Böhle M, Haub S, et al. (2013) Role of serotonin in fatty acid-induced non-alcoholic fatty liver disease in mice. BMC Gastroenterol 13, 169.

52. Spruss A \& Bergheim I (2009) Dietary fructose and intestinal barrier: potential risk factor in the pathogenesis of nonalcoholic fatty liver disease. $J$ Nutr Biochem 20, 657-662.

53. Hold GL (2014) Western lifestyle: a 'master' manipulator of the intestinal microbiota? Gut 63, 5-6.

54. De Bandt J-P, Waligora-Dupriet A-J \& Butel M-J (2011) Intestinal microbiota in inflammation and insulin resistance: relevance to humans. Curr Opin Clin Nutr Metab Care 14, 334-340.

55. Mouzaki M, Comelli EM, Arendt BM, et al. (2013) Intestinal microbiota in patients with nonalcoholic fatty liver disease. Hepatology 58, 120-127.

56. Raman M, Ahmed I, Gillevet PM, et al. (2013) Fecal microbiome and volatile organic compound metabolome in obese humans with nonalcoholic fatty liver disease. Clin Gastroenterol Hepatol 11, 868-875.

57. Mazmanian SK, Liu CH, Tzianabos AO, et al. (2005) An immunomodulatory molecule of symbiotic bacteria directs maturation of the host immune system. Cell $\mathbf{1 2 2}$, 107-118.

58. Everard A, Belzer C, Geurts L, et al. (2013) Cross-talk between Akkermansia muciniphila and intestinal epithelium controls diet-induced obesity. Proc Natl Acad Sci U S A 110, 9066-9071.

59. Zhang X, Shen D, Fang Z, et al. (2013) Human gut microbiota changes reveal the progression of glucose intolerance. PLOS ONE 8, e71108. 
60. Morizono H, Cabrera-Luque J, Shi D, et al. (2006) Acetylornithine transcarbamylase: a novel enzyme in arginine biosynthesis. J Bacteriol 188, 2974-2982.

61. Xu Y, Labedan B \& Glansdorff N (2007) Surprising arginine biosynthesis: a reappraisal of the enzymology and evolution of the pathway in microorganisms. Microbiol Mol Biol Rev 71, $36-47$.

62. Noack J, Kleessen B, Proll J, et al. (1998) Dietary guar gum and pectin stimulate intestinal microbial polyamine synthesis in rats. J Nutr 128, 1385-1391.
63. Mong M, Chao C \& Yin M (2011) Histidine and carnosine alleviated hepatic steatosis in mice consumed high saturated fat diet. Eur J Pharmacol 653, 82-88.

64. Senthilkumar R, Viswanathan P \& Nalini N (2003) Glycine modulates hepatic lipid accumulation in alcohol-induced liver injury. Pol J Pharmacol 55, 603-611.

65. Caldow MK, Ham DJ, Godeassi DP, et al. (2015) Glycine supplementation during calorie restriction accelerates fat loss and protects against further muscle loss in obese mice. Clin Nutr (Epublication ahead of print 22 September 2015). 